\title{
CRISPR/Cas9-engineered Gad1 elimination in rats leads to complex behavioral changes: implications for schizophrenia
}

\author{
Kazuyuki Fujihara $\mathbb{D}^{1,2}$, Kazuo Yamada ${ }^{3}$, Yukio Ichitani ${ }^{3}$, Toshikazu Kakizaki ${ }^{1}$, Weiru Jiang ${ }^{1}$, Shigeo Miyata', \\ Takashi Suto $\mathbb{1 0}^{4}$, Daiki Kato ${ }^{4}$, Shigeru Saito ${ }^{4}$, Masahiko Watanabe ${ }^{5}$, Yuki Kajita ${ }^{6}$, Tomokazu Ohshiro ${ }^{6}$, \\ Hajime Mushiake $\mathbb{1 0}^{6}$, Yoshiki Miyasaka $\mathbb{1}^{7}$, Tomoji Mashimo ${ }^{7,8}$, Hiroki Yasuda $\mathbb{1 0}^{9}$ and Yuchio Yanagawa
}

\begin{abstract}
GABAergic dysfunctions have been implicated in the pathogenesis of schizophrenia, especially the associated cognitive impairments. The GABA synthetic enzyme glutamate decarboxylase 67-kDa isoform (GAD67) encoded by the GAD1 gene is downregulated in the brains of patients with schizophrenia. Furthermore, a patient with schizophrenia harboring a homozygous mutation of GAD1 has recently been discovered. However, it remains unclear whether loss of function of GAD1 leads to the symptoms observed in schizophrenia, including cognitive impairment. One of the obstacles faced in experimental studies to address this issue is the perinatal lethality of Gad1 knockout (KO) mice, which precluded characterization at the adult stage. In the present study, we successfully generated Gad1 KO rats using CRISPR/Cas9 genome editing technology. Surprisingly, 33\% of Gad1 KO rats survived to adulthood and could be subjected to further characterization. The GABA concentration in the Gad1 KO cerebrum was reduced to 52\% of the level in wild-type rats. Gad1 KO rats exhibited impairments in both spatial reference and working memory without affecting adult neurogenesis in the hippocampus. In addition, Gad1 KO rats showed a wide range of behavioral alterations, such as enhanced sensitivity to an NMDA receptor antagonist, hypoactivity in a novel environment, and decreased preference for social novelty. Taken together, the results suggest that Gad1 KO rats could provide a novel model covering not only cognitive deficits but also other aspects of the disorder. Furthermore, the present study teaches an important lesson: differences between species should be considered when developing animal models of human diseases.
\end{abstract}

\section{Introduction}

$\gamma$-Aminobutyric acid (GABA) is a major inhibitory neurotransmitter in the mammalian central nervous system. GABA is synthesized from glutamate by two glutamate decarboxylases (GADs), 67 and 65-kDa isoforms (GAD67 and GAD65) encoded by Gad1 and Gad2 genes, respectively ${ }^{1-3}$. While GAD67 is constitutively active and

\footnotetext{
Correspondence: Kazuyuki Fujihara (psy_fujihara@gunma-u.ac.jp) or Yuchio Yanagawa (yuchio@gunma-u.ac.jp)

'Department of Genetic and Behavioral Neuroscience, Gunma University Graduate School of Medicine, Maebashi City, Gunma 371-8511, Japan

${ }^{2}$ Department of Psychiatry and Neuroscience, Gunma University Graduate School of Medicine, Maebashi City, Gunma 371-8511, Japan

Full list of author information is available at the end of the article
}

accounts for basal GABA synthesis in the soma of neurons, GAD65 is transiently activated and responsible for on-demand production in the axonal terminal ${ }^{4,5}$. The difference in phenotypes between knockout (KO) mice of the two GADs also has indicated distinct physiological roles: the Gad1 KO mice displayed cleft palate accompanied by perinatal lethality ${ }^{6}$, while the Gad $2 \mathrm{KO}$ mice developed epileptic seizures in adulthood ${ }^{7}$.

Deficits in GABA production could also have a large impact on brain function in humans. In the cerebral cortex of subjects with schizophrenia, GAD67 $\mathrm{mRNA}^{8-11}$ and proteins ${ }^{12}$ are decreased. Conversely, GAD65 expression is unaffected $^{10}$ or slightly reduced ${ }^{13}$. Interestingly, a study 
reported that GAD65 expression levels were reduced in schizoaffective disorder, but not in schizophrenia ${ }^{14}$. GAD67 downregulation is likely brain-wide because it was observed in the prefrontal cortex, parietal cortex, visual cortex $^{15}$, and hippocampus ${ }^{16}$. In schizophrenia, cognitive impairments such as working memory deficits are key symptoms affecting the functional outcome of patients ${ }^{17}$. Because the GABAergic system plays an important role in performing working memory tasks by synchronizing neuronal activity by generating gamma oscillation ${ }^{18}$, GAD67 reduction is hypothesized to be a cause of cognitive impairment in schizophrenia. Accordingly, GABA concentrations in the dorsolateral prefrontal cortex have been correlated with working memory performance in healthy humans ${ }^{19}$.

Genetic evidence has also supported the link between the GAD1 gene and schizophrenia. Some single nucleotide polymorphisms (SNPs) surrounding the GAD1 locus have been associated with childhood-onset schizophrenia in a North American cohort ${ }^{20}$ and in ordinary schizophrenia in a Chinese cohort ${ }^{21}$. Among these SNPs, one was significantly associated with lower levels of GAD67 expression in postmortem analysis ${ }^{22}$. Some SNP variations in GAD1 were also associated with the poorer performance of attention and working memory ${ }^{23}$. Furthermore, homozygous missense mutations in the GAD1 gene were identified in a family of schizophrenia patients by whole-exome sequencing in Italy ${ }^{24,25}$. This mutation prevents the homodimerization of GAD67 proteins, which is necessary for their sufficient activity $^{25}$. Based on these genetic findings combined with accumulated evidence from postmortem brain studies, we can assume that loss-of-function mutations of Gad1 have a causal effect on the symptoms of schizophrenia, particularly on cognitive impairment. However, because the above studies are observational studies, it is unclear whether the Gad1 mutation is the cause of the disorder.

The development of a Gad1 knockout (KO) animal and characterization of its behavior is a reasonable strategy to test this hypothesis. However, since global Gad1 KO is lethal in mice on the first postnatal day ${ }^{6,26}$, they cannot undergo behavioral testing. Researchers have also developed several conditional KO mice or knockdown (KD) mice in which GAD67 was disrupted in a restricted subpopulation of GABAergic neurons to avoid lethality ${ }^{27-29}$. However, these mice never exhibited any working memory impairment despite showing a few schizophreniarelated phenotypes. Do these results ultimately refute the "GAD67 hypothesis" of cognitive impairments in schizophrenia?

Animal species used for $\mathrm{KO}$ experiments in biomedical research have been largely restricted to mice due to technical limitations. Recently, genome editing techniques such as the clustered regularly interspaced short palindrome repeat (CRISPR)/Cas9 system $^{30}$ have allowed researchers to develop $\mathrm{KO}$ or transgenic animals in various species other than mice, such as rats ${ }^{31,32}$, ferrets ${ }^{33}$, and marmosets ${ }^{34}$. In this situation, careful examination is necessary when selecting a species suitable for experiments ${ }^{35}$. Although both mice and rats belong to the order Rodentia, there are significant differences that can affect behavioral testing. First, most behavioral tests were originally developed in rats, and some of these tests are more suitable for rats than mice. For instance, in the Morris water maze test used for the assessment of spatial memory, mice have greater difficulty in learning the location of the platform compared to rats because mice tend to avoid swimming as a habit ${ }^{35}$. Second, rats are suggested to have a much abundant repertoire of behavioral tests for investigating cognitive functions, which is advantageous for research on psychiatric disorders ${ }^{31}$. This can make a difference in detecting possible cognitive impairments of Gad1 knockout animals. In addition, with respect to GAD67 and GAD65 expression levels, a species difference has already been reported. We have previously shown that the expression levels of GAD67 and GAD65 are similar in the adult mouse brain ${ }^{28}$. Conversely, although the biological significance is unclear, the expression level of GAD67 is lower than that of GAD65 in rat and human brains ${ }^{36,37}$. Thus, the GAD67/GAD65 expression ratio in human brains is closer to that in rat brains than in mouse brains, suggesting that the rat is an invaluable experimental animal for studying the roles of GAD67 in brain function and dysfunction, in particular, the pathophysiology of schizophrenia.

Based on the above, we planned to re-examine the phenotypes caused by global and conditional Gad1 KO in rats rather than mice. Surprisingly, we found that some global Gad1 KO rats can survive to adulthood. Therefore, herein, we focused on the behavioral characterization of global Gad1 KO rats to provide evidence supporting the cause-effect relationship between loss of function of GAD67 and schizophrenia-related phenotypes, including cognitive impairment. Our data will provide insight into not only the pathophysiology of the patient with the ultrarare mutation of GAD1 but also that of other ordinary patients.

\section{Materials and methods}

All experiments were approved by the Animal Care and Experimentation Committees of Gunma University, the Animal Research Committee of Osaka University, and the Institutional Laboratory Animal Care and Use Committee of Tohoku University. Every effort was made to minimize the number of animals used and their suffering.

\section{Animals}

A rat line with exon-6 deletion of Gad1 (Gene ID: 24379) was generated on a Long-Evans background 


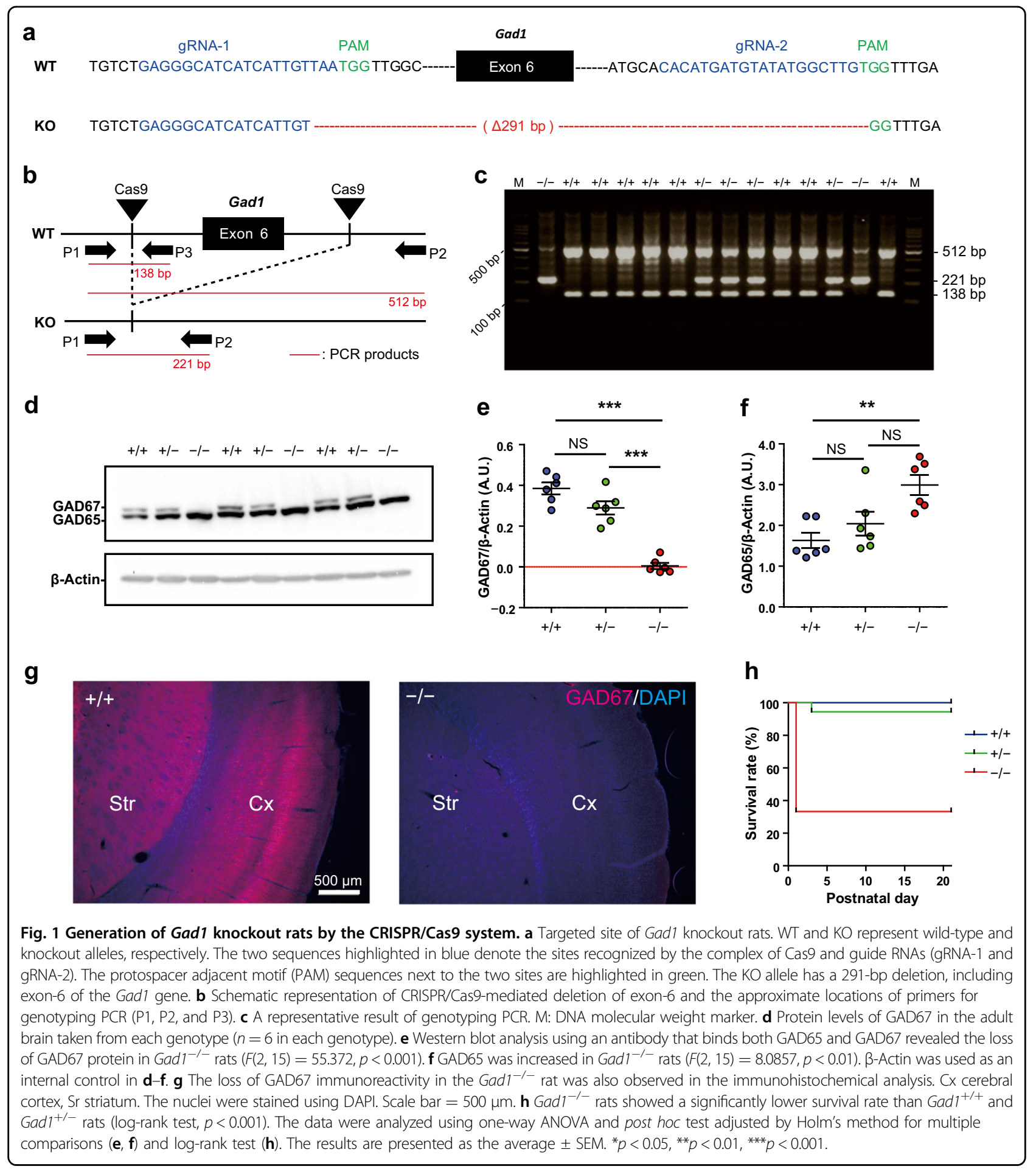

(Japan SLC, Inc., Hamamatsu, Shizuoka, Japan) using previously described methods ${ }^{38}$. It is noteworthy that rat Gad1 exon-6 corresponds to mouse Gad1 exon- $5^{39,40}$. Briefly, two CRISPR guide RNAs (gRNAs) were designed to target exon-6 of Gad1 (Fig. 1a). To obtain both Gad1 $\mathrm{KO}$ and Gad1-flox lines, a long single-strand DNA
(lssDNA) composed of exon-6 flanked by two loxP sequences was electroporated with the two gRNAs and Cas9 mRNA into pronuclear stage embryos. The Gad1flox line will be described elsewhere; herein, we focused on the Gad1 KO line. Embryos developing into the twocell stage after the introduction of RNAs and lssDNA 
were transplanted into oviducts of foster mothers. We successfully acquired an F0 rat with a $291 \mathrm{bp}$ deletion, which includes exon-6 (Fig. 1a). The F0 rat was crossed with wild-type (WT) Long-Evans rat to obtain F1 heterozygous $\mathrm{KO}$ rats. For further experiments, we crossed heterozygous male and female rats to obtain WT $\left(\mathrm{Gad1}^{+/+}\right)$, heterozygous $\left(\mathrm{Gadl}^{+/-}\right)$, and homozygous $\left(\mathrm{Gad1}^{-/-}\right)$ animals. The rats were housed in a room maintained at $22 \pm 3{ }^{\circ} \mathrm{C}$ with a 12 -h light-dark cycle (lights on at 6:00, lights off at 18:00). Food (CLEA Rodent Diet CE-2, Clea Japan, Meguro, Tokyo, Japan) and water were provided ad libitum.

\section{Genotyping PCR}

The genotype of each obtained pup was determined using genomic DNA extracted from the tail tissue at P0 (to determine the survival curve) or P21-P28 (for other experiments). PCR genotyping was performed using the following primers: 5'-ACTGGGCCATTGTTCCAGCTC CA-3' (primer 1), 5'-GCTCTCTCACGAGTATGCCCTT GCT-3' (primer 2), and 5'-CGAGCTGGAGAAGGGGG AAGAAGAT-3' (primer 3). Two DNA fragments of $512 \mathrm{bp}$ (primer 1 and primer 2) and $138 \mathrm{bp}$ (primer 1 and primer 3) were amplified from the WT allele, and a fragment of $221 \mathrm{bp}$ (primer 1 and primer 2) was amplified from the $\mathrm{KO}$ allele (Fig. 1b, c).

\section{Quantification of GAD proteins and GABA in rat brain tissue}

Elimination of GAD67 in Gad1 KO rats was confirmed in cerebral cortex tissue samples taken from adult (three months old) or juvenile rats (P20-P24). GAD67 or GAD65 protein was quantified by western blot analysis as described previously ${ }^{28}$. Concentrations of GABA and glutamate were measured by high-pressure liquid chromatography (HPLC) ${ }^{41}$ in the cerebral cortex and whole cerebellum samples of rats at P20-P24 (Supplementary Information).

\section{Immunohistochemistry}

Perfusion with $4 \%$ paraformaldehyde and immunohistochemistry were performed as described in the Supplementary materials using mouse anti-GAD67 (1:1000; Merck Millipore, Burlington, MA, USA) and antidoublecortin (DCX) antibodies (1:1000; Merck Millipore). See Supplementary Information for details.

\section{Behavioral analysis}

Before the behavioral tests, rats were habituated to the experimenters by a 3-day handling period. All rats were male and older than 10 weeks at the start of the behavioral analyses. We performed the Morris water maze, eight-arm radial maze, open field, novel object recognition, social interaction, Y-maze, elevated plus maze, acoustic startle response, prepulse inhibition (PPI), and forced swim tests. The eight-arm radial maze test was performed as described previously with minor modifications ${ }^{42}$. For the Morris water maze test, we modified the method of Nunez ${ }^{43}$. All other behavioral tests were carried out using a modified version of Fujihara et al. ${ }^{28}$ for rats. Except in the Morris water maze and radial maze tests, the genotypes of each rat were blinded to the experimenter. See the Supplementary Information for detailed procedures.

\section{Statistical analysis}

The sample sizes of each experiment were determined based on our previous study ${ }^{28}$. No randomization was performed. For comparison between genotypes, we employed Welch's $t$-test, Wilcoxon rank-sum test, oneway or two-way ANOVA with post hoc Holm's method. Although the variances of each group were similar in most of the experiments, we applied the Welch's $t$-test rather than Student's $t$-test because the former is more robust to unequal variances. If the Shapiro-Wilk normality test showed a lack of normality of the data, we applied the Wilcoxon rank-sum test for group comparison. To analyze the correlations between these parameters, we calculated Spearman's rank correlation coefficient $(r h o)$. For survival analysis, we used the Kaplan-Meier method with a log-rank test. We also conducted an analysis of covariance (ANCOVA) to compare the number of DCX-positive cells between the groups to adjust for age effects. $p$-values $<0.05$ were considered statistically significant.

\section{Results}

\section{Verification of the Gad1 knockout}

We successfully obtained a Gad1 KO rat line, as shown in Fig. 1a-c and Supplementary Fig. 1a, b. To verify that $\mathrm{Gad1}^{-1-}$ rats expressed no GAD67 protein, we carried out Western blot analysis for the brain extract using two different antibodies (Fig. 1d and Supplementary Fig. 1b). GAD67 protein was undetectable in the $\mathrm{Gad1}^{-1-}$ rats, whereas the amount of GAD65 protein in $\mathrm{Gad1}^{-1-}$ rats was significantly upregulated to $183.5 \%$ of that in $\mathrm{Gad} 1^{+/+}$ rats (Fig. 1f). A slight reduction in GAD67 protein in $\mathrm{Gad1}^{+/-}$rats was observed but did not reach statistical significance. The elimination of GAD67 was further confirmed by immunohistochemistry in the cerebral cortex and striatum. No immunoreactivity was detected in Gad1 $^{-/-}$rats (Fig. 1g).

\section{Lower survival rate and growth delay of $\mathrm{Gad1}^{-/-}$rats}

Each genotype of the rats was obtained at a Mendelian frequency (Supplementary Fig. 1a). The survival rate of Gad1 $1^{-/-}$rats was significantly lower than that of $\mathrm{Gad1} 1^{+/+}$ littermates, but $33 \%$ of $\mathrm{Gad1}^{-1-}$ rats grew to adulthood (Fig. 1h). The survival rate of $G a d 1^{+/-}$rats was not 
significantly different from that of $\mathrm{Gad1}^{+/+}$rats. This result was in contrast to cases of $\mathrm{Gad1}^{-1-}$ mice with $100 \%$ lethality at $\mathrm{P}^{6}$. Furthermore, no apparent malformations, such as the cleft palate or omphalocele, were observed in $\mathrm{Gad1}^{-1-}$ mice $^{6,26}$. Gad1 $1^{-1-}$ rats did not show any hindlimb clasping, suggesting that they had no ataxic-like motor dysfunction (Supplementary Fig. 1c). Conversely, the body size and body weight of $\mathrm{Gad1}^{-1-}$ rats were smaller than those of $\mathrm{Gad} 1^{+/+}$rats after birth to approximately two months of age (P28: Gad1 ${ }^{+/+}, 92.5 \pm$ 2.96 g; Gad1 $1^{-/-}, 59.65 \pm 10.23$ g; Supplementary Fig. 2), although the growth curve of homozygous $\mathrm{Gad1}^{-1-}$ rats caught up to adult rats (Supplementary Fig. 2c, d). Furthermore, the brains of $\mathrm{Gad1}^{-1-}$ rats had no obvious abnormality in gross morphology at the adult stage compared with $\mathrm{Gad1}^{+/+}$rats (data not shown).

\section{Decrease in GABA concentration in $\mathrm{Gad}^{-1-}$ rats}

The functional impact of GAD67 deletion in $\mathrm{Gad1}^{-/-}$ rats was measured by the concentration of GABA in brain tissue (Fig. 2). GABA concentrations in both the cerebral cortex and cerebellum were significantly reduced in homozygous $G a d 1^{-1-}$ rats (Fig. 2a, d), while $G a d 1^{+/-}$rats showed no significant reductions. Concentrations of glutamate (Glu), the precursor of GABA, showed no differences among the three genotypes in either region (Fig. 2b, e).
The GABA/Glu ratio differed among all genotypes in both the cerebral cortex and cerebellum (Fig. 2c, f).

\section{Reduced locomotion and rearing in $\mathrm{Gad1}^{-1-}$ rats}

Behavioral consequences of Gad1 deletion were determined by behavioral testing in $\mathrm{Gad1}^{-/-}$rats and $\mathrm{Gad1} 1^{+/+}$ littermates. In the open field test for seven days, Gad1 $1^{-1-}$ rats showed reduced distance traveled compared with $\mathrm{Gad1}^{+/+}$rats (Fig. 3a), although their moving speed was comparable to $\mathrm{Gad1}^{+/+}$rats (Fig. $3 \mathrm{~b}$ ). The duration per movement and the number of rearing events were also reduced in $\mathrm{Gad1}^{-1-}$ rats (Fig. 3c, d). Figure 3 reports the 7day summations for each parameter. The time course of each parameter is shown in Supplementary Fig. 3. As a whole, $\mathrm{Gad1}^{-1-}$ rats were characterized by hypoactive behavior.

The exploration time in the center region (center time) and number of fecal boli in the open field were measured. Because the data of the exploration time in the center region (center time) in $\mathrm{Gad1}^{-1-}$ rats showed neither normality ( $W=0.845, p<0.05)$ nor equality of variance to $\operatorname{Gad1}^{+/+}$rats $(F(11,11)=9.1871, p<0.001)$, a nonparametric test was used for comparisons. There was a significant decrease in the center time in $\mathrm{Gad1}^{-1-}$ rats (Fig. 3e; Supplementary Fig. 4a). The total number of fecal boli in the open field session also increased in $\mathrm{Gad1}^{-1-}$ rats (Supplementary Fig. 4b) and negatively correlated with
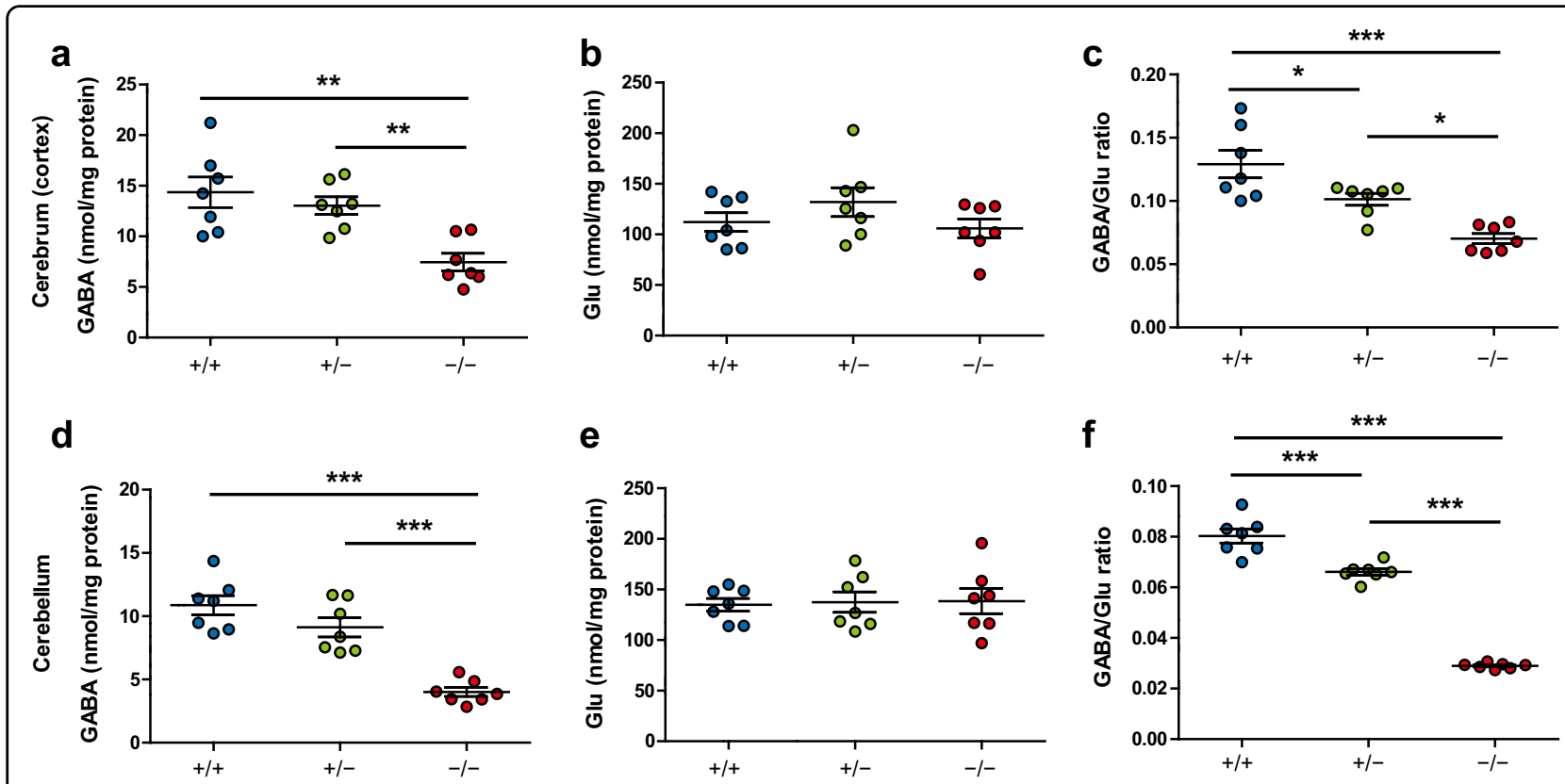

Fig. 2 GABA and glutamate concentrations in the brains of Gad 1 knockout rats. Brain samples were collected from P20 to P24. a In the cerebral cortex, the GABA concentration in Gad1 ${ }^{-1-}$ rats was reduced to $51.88 \%$ of that of $\mathrm{Gad}^{+/+}(F(2,18)=16.748, p<0.001)$. $\mathbf{b}$ There were no differences in the glutamate (Glu) concentrations among the three genotypes in the cerebral cortex $(F(2,18)=1.4734, p=0.2555)$. c The GABA/Glu ratio was reduced in both $\mathrm{Gad1}^{+/-}$and $\mathrm{Gad1}^{-1-}$ rats in the cerebral cortex $(F(2,18)=10.578, p<0.001)$. d GABA concentrations in the cerebellum $(F(2,18)=$ 29.646, $p<0.001)$. e Glu concentrations in the cerebellum $(F(2,18)=0.0372, p=0.9636)$. $\mathbf{f} \mathrm{GABA} / \mathrm{Glu}$ ratios in the cerebellum $(F(2,18)=218.79$, $p<0.001) . n=7$ in each genotype. The results are presented as the average \pm SEM. The data were analyzed using one-way ANOVA and post hoc test adjusted by Holm's method for multiple comparisons. ${ }^{*} p<0.05,{ }^{* *} p<0.01,{ }^{* * *} p<0.001$. 


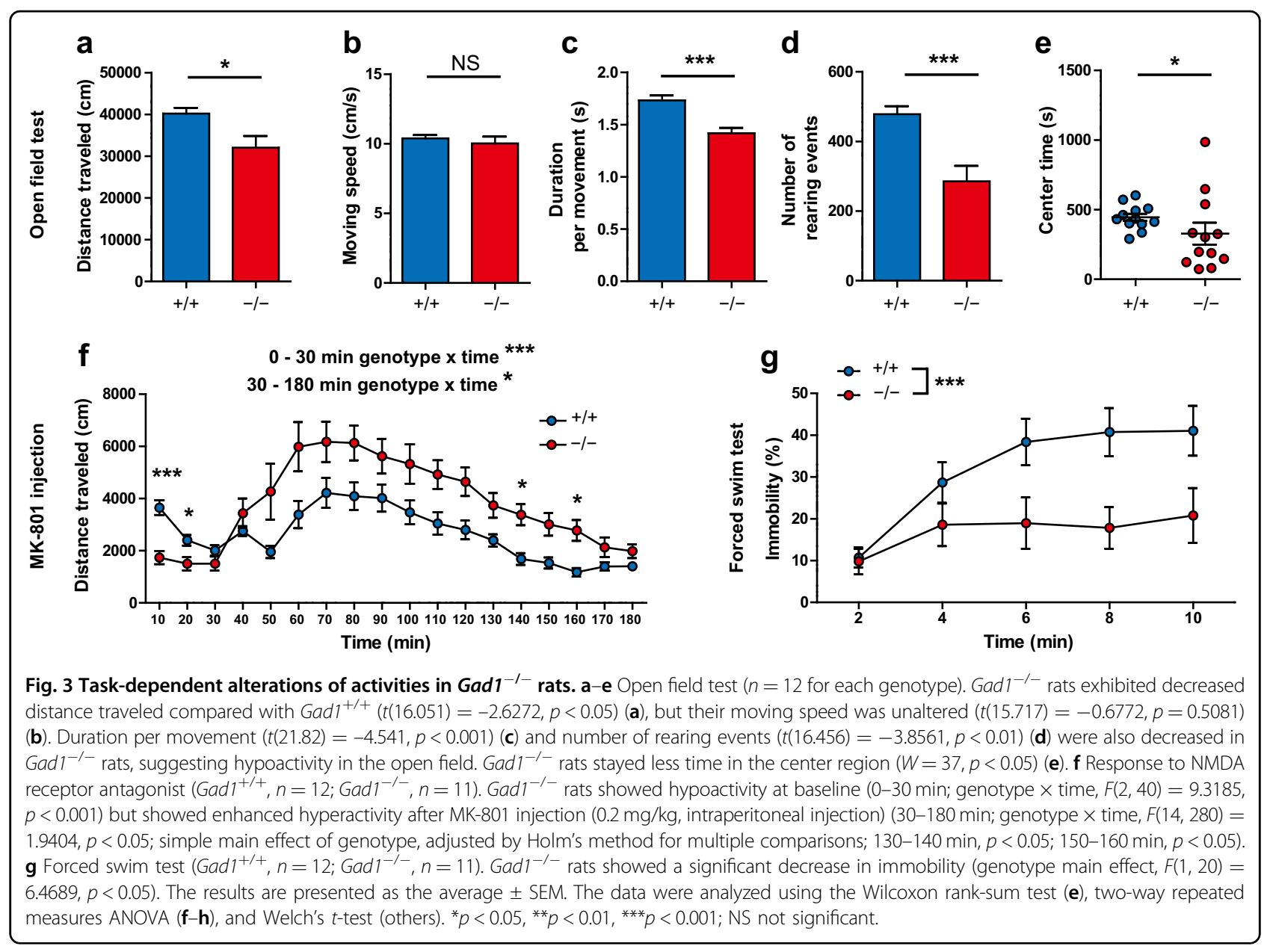

the center time and distance traveled only in Gad1 ${ }^{-1-}$ rats (Supplementary Fig. 4c, d).

Gad1 ${ }^{-1-}$ rats showed no alterations in the elevated plusmaze test

We assessed the behavior of $\mathrm{Gad1}^{-1-}$ rats on an elevated plus-maze. However, we did not detect any alterations, such as reduced exploration of the open arms of the maze (Supplementary Fig. 5).

\section{Enhanced sensitivity to NMDA receptor antagonists in $\mathrm{Gad1}^{-1-}$ rats}

Treatment with NMDA receptors can elicit schizophrenia-like symptoms in humans and schizophrenialike behaviors in rodents ${ }^{28,44}$. Therefore, we analyzed the sensitivity to the NMDA antagonist MK-801 $(0.2 \mathrm{mg} / \mathrm{kg}$, intraperitoneal injection) (Fig. 3f). During the habituation phase of the open field arena before administration of MK801, Gad1 $1^{-1-}$ rats showed significantly lower locomotor activity. Following MK-801 injection, the moving distance in both genotypes increased from baseline. Meanwhile, the distance traveled by $\mathrm{Gad1}^{-/-}$rats was significantly higher than that traveled by $G a d 1^{+/+}$rats. Subsequent post hoc tests confirmed the enhanced hyperlocomotion induced by MK-801 in $\mathrm{Gad1}^{-/-}$rats compared with $\mathrm{Gad1} 1^{+/+}$rats.

\section{Gad1 ${ }^{-I-}$ rats exhibited reduced immobility in the forced swim test}

Gad1 $1^{-1-}$ rats also showed enhanced activity in the forced swim test. In the test session, $\mathrm{Gad} 1^{-1-}$ rats became significantly less immobile than $\mathrm{Gad} 1^{+/+}$rats (Fig. 3g).

\section{Normal acoustic startle response and sensorimotor gating in $\mathrm{Gad1}^{-1-}$ rats}

Deficits in sensorimotor gating are a translatable endophenotype of schizophrenia ${ }^{28}$. We tested possible deficits in the startle response and sensorimotor gating using an acoustic startle response, yet there were no differences between $\mathrm{Gad1}^{-/-}$and $\mathrm{Gad1} 1^{+/+}$rats (Supplementary Fig. 6).

\section{Reduced social novelty preference in $\mathrm{Gad}^{-1-}$ rats with intact object recognition}

We assessed the short-term recognition memory of Gad $1^{-1-}$ rats using the novel object recognition task ${ }^{45}$. 


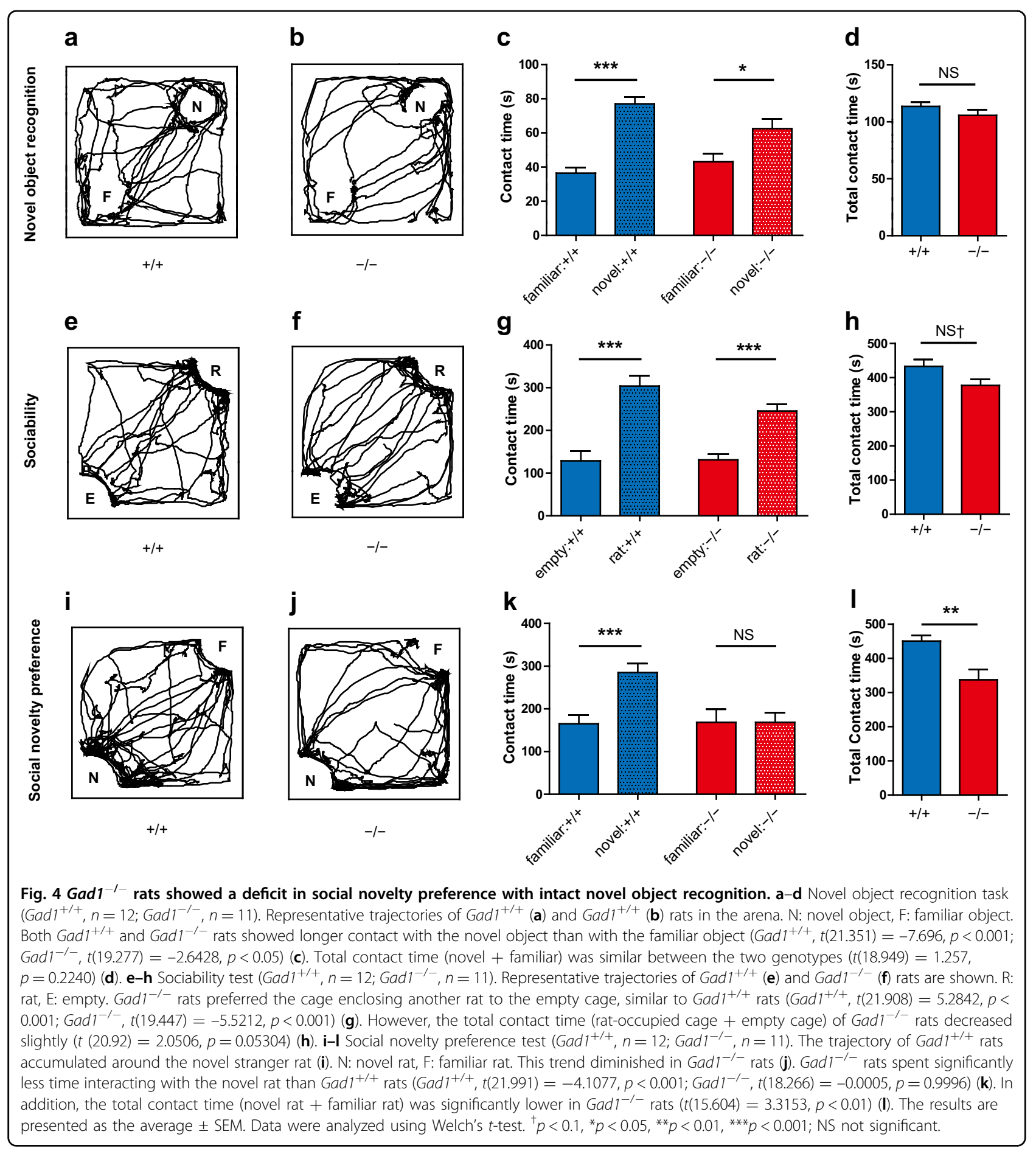

Both $\mathrm{Gad1}^{-/-}$and $\mathrm{Gad1}^{+/+}$rats explored the novel object longer than the familiar object (Fig. $4 a-c)$. The total contact time to the objects (novel object + familiar object) was also comparable between the two genotypes (Fig. 4d). Thus, Gad1 ${ }^{-1-}$ rats had no obvious deficit in recognition memory, at least discriminating between two objects.
We also tested rats for sociability and social novelty preference using the same apparatus as the novel object recognition test. In the sociability test for social vs. empty preferences, both genotypes of rats spent more time interacting with the rat-occupied cage than with the empty cage (Fig. $4 \mathrm{e}-\mathrm{g}$ ). Gad1 $1^{-1-}$ rats showed slightly reduced total contact times (rat-occupied cage + empty cage) but at 
a subthreshold level (Fig. 4h). Subsequently, we examined social novelty preferences, which are relevant to social memory. While $\mathrm{Gad1}^{+/+}$rats spent more time interacting with the novel rat than with the familiar rat (Fig. 3i, k), Gad1 ${ }^{-1-}$ rats did not display such a preference (Fig. 4 j-1). Furthermore, $\mathrm{Gad1}^{-1-}$ rats showed significantly reduced total contact times (novel rats + familiar rats) (Fig. 4l).

\section{Gad1 $1^{-1-}$ rats exhibited spatial cognitive impairments and aberrant hyperactivity}

To assess the spatial reference memory of $\mathrm{Gad} 1^{-1-}$ rats, the hidden platform task in the Morris water maze was used. During task training, the latency to escape of $\mathrm{Gad} 1^{-1-}$ rats was significantly longer than that of $\mathrm{Gad1} 1^{+/+}$rats (Fig. 5a). However, the swimming speed of $\mathrm{Gad1}^{-1-}$ rats was unaltered (Fig. 5b). Furthermore, in the probe test the day after the end of the training, $\mathrm{Gad1} 1^{-/-}$rats stayed less in the target quadrant (Fig. $5 \mathrm{c}-\mathrm{e}$ ). In the visible platform test, however, there was no significant difference in the escape latency between the two genotypes (Fig. 5f).

Spatial working memory was measured using the Y-maze task (Fig. 5g, h) and the eight-arm radial maze task (Fig. 5i-k). Gad1 ${ }^{-1-}$ rats showed reduced spontaneous alternation behavior in the Y-maze task (Fig. 5g). Total entry number to arms, an indicator of locomotor activity on the maze, showed a trend of reduction in $G a d 1^{-1-}$ rats, albeit the difference was not statistically significant (Fig. 5h).

In the acquisition curve of the eight-arm radial maze task, there was a significant genotypextrial interaction on the number of correct choices (Fig. 5i). The post hoc test revealed that the performance of $\mathrm{Gad1}^{-1-}$ rats was significantly poorer than that of WT rats during the 7th to 10th block of training. The number of trials needed to meet the learning criterion (correct choice $\geq 7$ for five consecutive trials) was also prolonged (Fig. 5j). Conversely, $\mathrm{Gad1}^{-1-}$ rats showed slightly higher scores on the 2nd block (Fig. 5i). However, this did not indicate that $\mathrm{Gad} 1^{-1-}$ rats performed well because their score was similar to the chance-level value of the radial maze task $(\sim 5.3)^{46}$. This was caused by increased running speed from the early stage of training in $\mathrm{Gad1}^{-1-}$ rats (see below). A similar acquisition curve was observed in a pharmacological model of schizophrenia in our previous study ${ }^{42}$. The running time per choice ${ }^{42}$, which reflects the running speed of rats during the task, was significantly shortened in $\mathrm{Gad} 1^{-1-}$ rats (Fig. 5k). The faster a rat runs on the maze, the smaller the running time becomes. Therefore, the results indicate that $\mathrm{Gad1} 1^{-1-}$ rats moved faster than $\mathrm{Gadl}^{+/+}$rats in the radial maze task, unlike in the open field test and the Y-maze task.

\section{Adult neurogenesis in the dentate gyrus was not altered in $\mathrm{Gad1}^{-1-}$ rats}

The results from the behavioral tests strongly suggest that $\mathrm{Gad1} 1^{-/-}$rats had spatial cognitive deficits, which can be affected by abnormalities in adult neurogenesis. GABAergic transmission plays a pivotal role in regulating adult neurogenesis ${ }^{47}$. Furthermore, alterations in adult neurogenesis have been reported in some animal models of schizophrenia ${ }^{48}$. DCX is known as one of the molecular markers of neurogenesis ${ }^{48}$. Therefore, we investigated possible impairments of adult neurogenesis in the hippocampus, where we counted DCX-positive cells in the dentate gyrus of $\mathrm{Gad1}^{-/-}$and $\mathrm{Gad1} 1^{+/+}$rats (Supplementary Fig. 7a, b). Both genotypes showed an agedependent reduction in the number of DCX-positive cells. After adjustment for age, there was no difference in the number of DCX-positive cells (Supplementary Fig. 7c).

\section{Discussion}

To test the hypothesis that loss-of-function mutation in the GAD1 gene leads to cognitive impairments observed in schizophrenia, we generated Gad1 $\mathrm{KO}$ rats using genome editing. Despite the relatively high neonatal mortality and transient growth retardation during the developmental stage, behavioral tests were feasible in adulthood, unlike $\mathrm{Gad1}^{-1-}$ mice ${ }^{6}$. In line with our hypothesis, we found significant impairments in both spatial reference and working memory in adult male Gad1 ${ }^{-1-}$ rats. To our knowledge, this is the first direct evidence that elimination or decrease in GAD67 expression can cause distinct impairments in spatial memory in particular. Since we also identified behavioral alterations alongside cognitive impairments, $\mathrm{Gad} 1^{-/-}$rats may recapitulate a broader range of symptoms of schizophrenia.

How does loss of GAD67 expression lead to spatial cognitive impairments? The Morris water maze, eightarm radial maze, and Y-maze tasks are known as hippocampus-dependent tasks ${ }^{49-51}$. Although adult neurogenesis plays distinct roles in hippocampus-dependent functions such as $\operatorname{cognition}^{52}$, we did not detect any reduction in adult-born neurons in the $\mathrm{Gad1} 1^{-1-}$ hippocampus. Thus, the poorer performance observed in these tasks must be independent of hippocampal adult neurogenesis. CA1 and hilar GABAergic neurons are required for spatial working memory and spatial reference memory, respectively ${ }^{53,54}$. Therefore, cognitive impairments in Gad $1^{-I-}$ rats may be partially explained by loss of GAD67 and subsequent impaired GABAergic transmission. However, conditional $\mathrm{KO}$ or $\mathrm{KD}$ (cKO/KD) of Gad1, which mainly targets parvalbumin (PV)-positive GABAergic neurons, causes no impairment of spatial working memory in mice ${ }^{27-29,55}$. PV neurons are the largest population of GABAergic neurons ${ }^{56,57}$ and are preferentially impaired in schizophrenia ${ }^{12}$. In mice, acute optogenetic suppression of PV neurons in the hippocampus impairs working memory ${ }^{53}$, which is consistent with the present study but is inconsistent with the results 


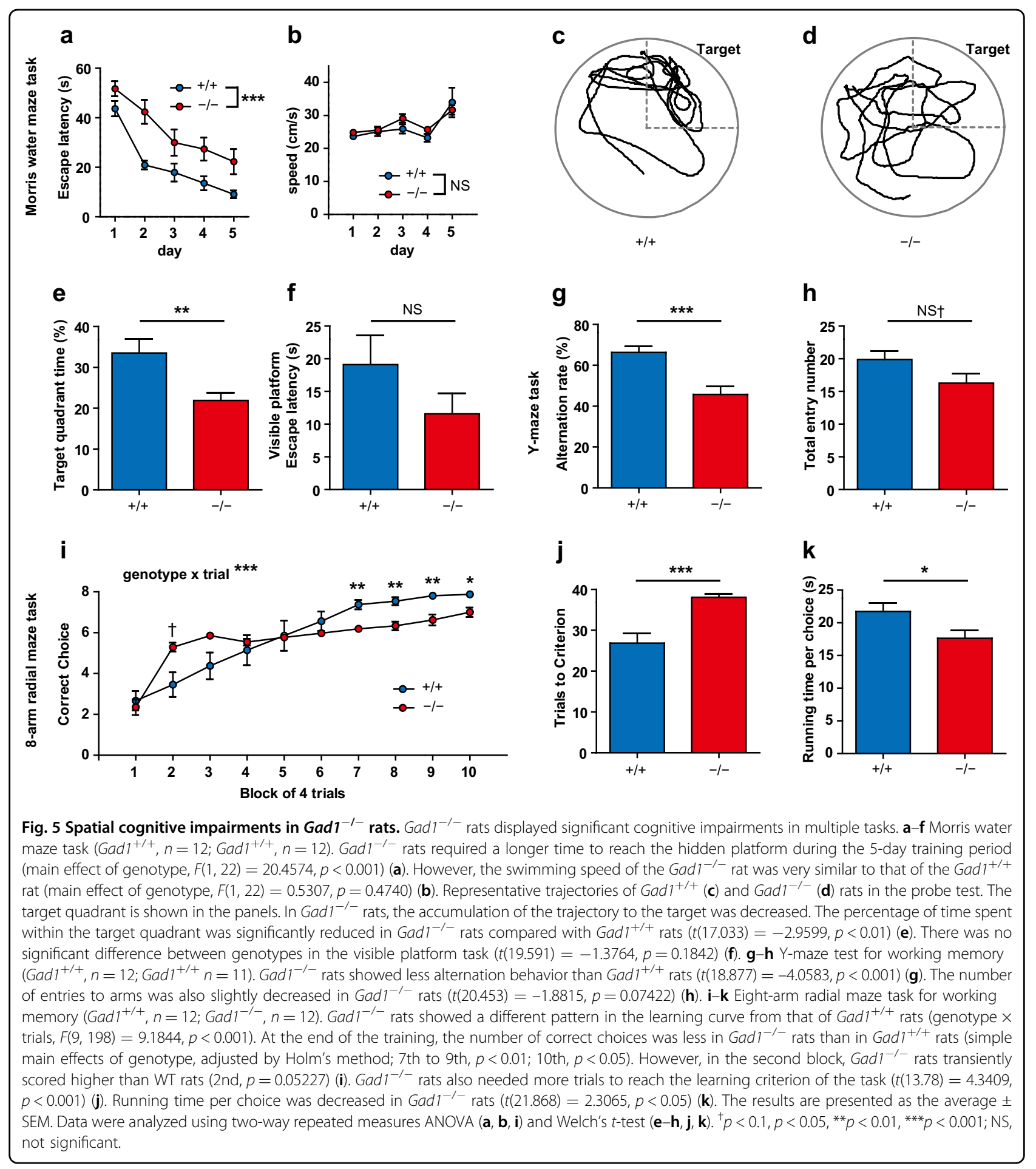

of $\mathrm{cKO} / \mathrm{KD}$ mice. The optogenetic technique probably can suppress GABAergic transmission more strongly than the genetic reduction of GAD67 because the release of GABA produced by GAD65 is also inhibited. Different effects on working memory between our global Gad1 KO rats and the $\mathrm{CKO} / \mathrm{KD}$ mice also may be attributed to differences in the severity of cognitive impairment. All previous studies of $\mathrm{cKO} / \mathrm{KD}$ mice employed only spontaneous alternation behavior on the Y-maze for evaluation of spatial working memory ${ }^{27-29,55}$. However, the number of arms to be memorized is considerably fewer in the Ymaze than in the eight-arm radial maze. In addition, spontaneous alternation is suggested to be less sensitive for detecting working memory impairment than the eight- 
arm radial maze task in some cases ${ }^{58}$. Compared to global $\mathrm{KO}$ rats, GAD67 reduction in $\mathrm{cKO} / \mathrm{KD}$ mice is considered to be mild. If the working memory impairment in the mice was also moderate, it would be difficult to detect it merely by spontaneous alternation. Furthermore, species differences in the functional role of GAD67 between mice and rats should also be considered as the cause of the discrepancy, although the significance of species differences in the GAD67/GAD65 ratio has yet to be determined. Gad1 cKO/KD rats are needed in the future to explore species differences more directly. Since we have generated a Gad1-flox rat line, this will be our focus in subsequent studies.

In addition to cognitive impairments, $\mathrm{Gad1}^{-1-}$ rats showed characteristic alterations in activity. In the open field test, spontaneous locomotion and rearing were significantly reduced, while NMDA receptor antagonist-induced hyperlocomotion was enhanced compared with $\mathrm{Gad1}^{+/+}$rats. We must carefully interpret the reduced activity in the context of schizophrenia research using animal models. One possible interpretation is that hypoactivity is a model of negative symptoms. Patients with chronic schizophrenia display increased resting time during daily activities, and the extent of their activity is negatively correlated with the severity of negative symptoms ${ }^{59}$. Even after the 7-day habituation period in the open field, $G a d 1^{-1-}$ rats still displayed a shorter duration of movement, i.e., a longer resting period. Therefore, the lower baseline activity of $\mathrm{Gad1}^{-1-}$ rats may represent a negative symptom-like phenotype. $\mathrm{Gad1}^{-1-}$ rats also displayed reduced social novelty preference, which is considered indicative of negative symptoms or impaired social recognition memory ${ }^{28}$. Because $\mathrm{Gad1}^{-1-}$ rats could perform normally in the novel object recognition task, this alteration is specific to the social context. Another speculation for hypoactivity is that it is a manifestation of increased anxiety in $\mathrm{Gad1}^{-1-}$ rats. We observed significant correlations among the center time, number of fecal boli, and locomotor activity in $\mathrm{Gad1}^{-1-}$ rats. The decrease in exploration time of the center region of the open field ${ }^{60}$ and increased number of fecal boli ${ }^{61}$ are considered anxiety-like behaviors in rodents. The enhanced anxiety-like phenotype is partially consistent with somatostatin neuron-specific Gad1 KO mice, which showed a decrease in the center time during the open field test ${ }^{62}$. Conversely, no differences between the two genotypes were noted in the elevated plus-maze test. Therefore, caution is warranted when making conclusions about anxiety levels at present.

In contrast to hypoactivity in the open field test, hypersensitivity to NMDA receptor antagonists is relatively easy to interpret as a schizophrenia symptom. This phenotype is considered a hallmark of animal models of positive symptoms ${ }^{28}$ and is suggested to be mediated by the dopaminergic system ${ }^{63}$. Therefore, our results suggest that the decrease in GAD67 levels also influences the positive symptom-like phenotype in rats. It will be of interest to determine whether dopamine levels are altered following the administration of an NMDA receptor antagonist or amphetamine in $G a d 1^{-1-}$ rats. Because this phenotype is shared with PV neuron-specific Gad1 heterozygous $\mathrm{KO}$ mice ${ }^{28}$, as we have reported previously, it is also likely to be mediated by dysfunction of this subtype of GABAergic neurons.

$\mathrm{Gad1}^{-1-}$ rats also share some phenotypes with pharmacological models of schizophrenia. Neonatal repetitive administration of NMDA receptor antagonists, such as MK-801 and ketamine, are pharmacological models of schizophrenia ${ }^{42,58,64,65}$. MK-801-treated rats showed significantly decreased rearing or a trend-level reduction in locomotor activity as adults, similar to $\mathrm{Gad1}^{-1-}$ rats $^{42,58,65}$. Surprisingly, these rats displayed hyperactivity in the eight-arm radial maze task $^{42}$ and decreased immobility in the forced swim test after stress ${ }^{58}$. Although the neurobiological basis of these behavioral manifestations in NMDA receptor antagonist-treated rats remains to be determined, some common mechanisms may exist between $\mathrm{Gad1}^{-1-}$ rats and NMDA receptor antagonisttreated rats. Notably, blockade of NMDA receptors during the postnatal period induces a marked reduction in PV-positive GABAergic neurons ${ }^{66}$ and GAD67 protein $^{67}$. Assuming that GABAergic dysfunction itself is the common pathway of the behavioral alterations, the $\mathrm{Gad1}^{-1-}$ rat model represents a helpful tool to reveal the downstream phenomena of repetitive postnatal NMDA receptor blockade.

This study also indicated that $\mathrm{Gad1}^{-1-}$ rats failed to show deficits in PPI, an intermediate phenotype in models of schizophrenia widely assessed in animal studies ${ }^{28,65}$. Because GAD65 elimination causes a robust deficit of PPI in mice ${ }^{68}$, GABAergic transmissions have been implicated in normal sensorimotor gating. However, interestingly, GAD67 elimination showed no alterations in PPI in rats. According to postmortem brain studies, GAD65 expression is unchanged in schizophrenia patients ${ }^{11}$, although we observed that GAD65 expression was markedly upregulated in $\mathrm{Gad1}^{-1-}$ rat brains. The intact PPI may be attributed to this molecular discrepancy between the patients and $\mathrm{Gad1}^{-/-}$rats.

Combined with studies on $\mathrm{Gad1}^{-1-}$ mice ${ }^{7}$, the present study revealed evident differences between mice and rats with loss-of-function mutations in Gad1. In terms of mortality, the $\mathrm{Gad1}^{-1-}$ phenotype in rats appears to be less severe than that of $\mathrm{Gad1}^{-1-}$ mice. Although the biological significance of the species difference in the GAD67/GAD65 ratio remains elusive, the reduced dependence on GAD67 in rats would enable them to survive to adulthood even if this isoform was lost. Furthermore, the cleft palate observed in $\mathrm{Gad1}^{-1-}$ mice was absent in $\mathrm{Gad1} 1^{-1-}$ rats, which implies 
that the lower GAD67/GAD65 ratio in rats prevented palate malformation as well. Meanwhile, we unexpectedly discovered a reduced body weight of $\mathrm{Gad1}^{-/-}$rats only during development. Although some evidence suggests that GABA has important roles in controlling food intake and secretion of growth hormone $e^{69-74}$, the precise mechanisms of growth retardation in $\mathrm{Gad1}^{-1-}$ rats should be addressed to exclude potential effects on brain development and behavior. Notably, GAD67 elimination reduced GABA levels to almost half those in $\mathrm{Gad1}^{+/+}$rat brains (Fig. 2), although the GAD67 protein amount accounted for only $23 \%$ of the total GAD proteins in the $\mathrm{Gad1}^{+/+}$rat brain ${ }^{36}$, and GAD65 showed compensatory upregulation in $\mathrm{Gad1}^{-1-}$ rats (Fig. 1f). These findings indicate that GAD67 is important for maintaining baseline GABA levels in rats. Asada et al. ${ }^{6}$ speculated that the cause of death in $\mathrm{Gad1}^{-/-}$ mice was respiratory failure rather than cleft palate. The lethality without cleft palate observed in a subpopulation of $\mathrm{Gad1}^{-1-}$ rats also supports their hypothesis.

We also need to acknowledge some limitations of the current study. First, the $\mathrm{Gad1} 1^{-1-}$ rats are just a model of an ideal situation wherein GAD67 is completely eliminated. As reported in the postmortem brain studies, GAD67 is not completely lost in schizophrenia. GAD67 mRNA levels are only reduced by $15-35 \%$ when compared to the unaffected population ${ }^{9,11,12}$. Moreover, this reduction occurs largely in the PV-positive GABAergic neurons ${ }^{12}$. The effect of milder reduction and cell typespecific knockout of GAD67, as well as their interaction with environmental factors, should be analyzed in future studies. Second, although some studies have discovered the association between GAD1 and schizophrenia, a recent genome-wide association study in a larger population did not report GAD1 as a susceptibility gene ${ }^{75}$. Therefore, it should be noted that the evidence of an association between the genetic variation of GAD1 and schizophrenia is limited. We also would like to emphasize that the $G a d 1^{-1-}$ rats are a model for revealing the effects of GAD67 reduction and not a model that exactly mimics the genetic variation of GAD1 discovered in patients.

In conclusion, the loss-of-function mutation in the Gad1 gene causes not only cognitive impairments but also several behavioral alterations possibly relevant to positive and negative symptoms of schizophrenia. Gad1 $1^{-/-}$rats will represent a novel tool to study pathophysiology and to develop treatments for cognitive impairment in schizophrenia. Furthermore, the results of our study warn researchers to pay closer attention to potential species differences between mice and rats when developing animal models of human disorders.

\section{Acknowledgements}

This work was supported by JSPS KAKENHI Grant Number JP26290002 (Y.Y.), JP15H05872 (Y.Y.), JP16H06276 [Platform of Advanced Animal Model Support, AdAMS] (Y.Y.), JP17H05550 (Y.Y.), JP17K17628 (K.F.), JP19K06881 (Y.Y.),
JP15H05879 (H.M.), and AMED under Grant Number JP19dm0207001 (H.M.). This project was also partly supported by the Takeda Science Foundation (Y.Y.), the Life Science Foundation of Japan (K.F.), and the Collaborative Research Project of the Brain Research Institute, Niigata University (Y.Y.). We are grateful to Takumi Sato, Misuzu Umehara, Shunsuke Teshima, Kazuya Higeta, Yugo Nakajima, Sota Abe, and Yuki Fukuda for technical assistance. We thank Dr. Ryosuke Kaneko for his helpful comments on the analysis of data and the members of our laboratory for their support and encouragement. We also thank the staff at the Institute of Experimental Animal Research, Gunma University Graduate School of Medicine. Finally, we thank Dr. Takako Fujihara for her encouragement and critical comments as a clinical psychiatrist.

\section{Author details}

'Department of Genetic and Behavioral Neuroscience, Gunma University Graduate School of Medicine, Maebashi City, Gunma 371-8511, Japan. ${ }^{2}$ Department of Psychiatry and Neuroscience, Gunma University Graduate School of Medicine, Maebashi City, Gunma 371-8511, Japan. ${ }^{3}$ Institute of Psychology and Behavioral Neuroscience, University of Tsukuba, Tsukuba City, Ibaraki 305-8577, Japan. ${ }^{4}$ Department of Anesthesiology, Gunma University Graduate School of Medicine, Maebashi City, Gunma 371-8511, Japan. ${ }^{5}$ Department of Anatomy, Faculty of Medicine, Hokkaido University, Sapporo City, Hokkaido 060-8638, Japan. ${ }^{6}$ Department of Physiology, Graduate School of Medicine, Tohoku University, Sendai City, Miyagi 980-8575, Japan. 'Institute of Experimental Animal Sciences, Graduate School of Medicine, Osaka University, Suita City, Osaka 565-0871, Japan. ${ }^{8}$ Laboratory Animal Research Center, Institute of Medical Science, The University of Tokyo, Minato-ku, Tokyo 108-8639, Japan. ${ }^{9}$ Division of Physiology, Faculty of Medicine, Saga University, Saga City, Saga 849-8501, Japan

\section{Author contributions}

K.F. and Y.Y. designed the research and wrote the manuscript. Y.M. and T.M. generated Gad1 KO rats. With help from T.K., S.M., T.S., D.K., S.S., T.O., H.M., M.W., and H.Y., Y.Y., K.F., W.J., K.Y., Y.I., and Y.K. performed all the other experiments. T.K. and M.W. produced antibodies. K.F., K.Y., Y.K., Y.I., and Y.Y. analyzed the data. All authors approved the final manuscript.

Conflict of interest

The authors declare that they have no conflict of interest.

\section{Publisher's note}

Springer Nature remains neutral with regard to jurisdictional claims in published maps and institutional affiliations.

Supplementary Information accompanies this paper at (https://doi.org/ 10.1038/s41398-020-01108-6).

Received: 22 June 2020 Revised: 6 November 2020 Accepted: 16 November 2020

Published online: 08 December 2020

\section{References}

1. Obata, K. Synaptic inhibition and $\gamma$-aminobutyric acid in the mammalian central nervous system. Proc. Jpn. Acad. Ser. B 89, 139-156 (2013).

2. Bu, D. F. et al. Two human glutamate decarboxylases, 65-kDa GAD and 67-kDa GAD, are each encoded by a single gene. Proc. Natl Acad. Sci. USA 89 2115-2119 (1992).

3. Soghomonian, J. \& Martin, D. L. Two isoforms of glutamate decarboxylase: why? Trends Pharmacol. Sci. 19, 500-505 (1998).

4. Patel, A. B., De Graaf, R. A., Martin, D. L., Battaglioli, G. \& Behar, K. L. Evidence that GAD65 mediates increased GABA synthesis during intense neuronal activity in vivo. J. Neurochem. 97, 385-396 (2006).

5. Kaufman, D. L., Houser, C. R. \& Tobin, A. J. Two forms of the $\gamma$-aminobutyricacid synthetic enzyme glutamate decarboxylase have distinct intraneuronal distributions and cofactor interactions. J. Neurochem. 56, 720-723 (1991).

6. Asada, H. et al. Cleft palate and decreased brain $y$-aminobutyric acid in mice lacking the 67-kDa isoform of glutamic acid decarboxylase. Proc. Natl Acad. Sci. USA 94, 6496-6499 (1997). 
7. Kash, S. F. et al. Epilepsy in mice deficient in the $65-\mathrm{kDa}$ isoform of glutamic acid decarboxylase. Proc. Natl Acad. Sci. USA 94, 14060-14065 (1997).

8. Hashimoto, T. et al. Gene expression deficits in a subclass of GABA neurons in the prefrontal cortex of subjects with schizophrenia. J. Neurosci. 23, 6315-6326 (2003).

9. Hashimoto, T. et al. Alterations in GABA-related transcriptome in the dorsolateral prefrontal cortex of subjects with schizophrenia. Mol. Psychiatry 13, 147-161 (2008).

10. Guidotti, A. et al. Decrease in reelin and glutamic acid decarboxylase67 (GAD67) expression in schizophrenia and bipolar disorder: a postmortem brain study. Arch. Gen. Psychiatry 57, 1061-1069 (2000).

11. Volk, D. W., Austin, M. C., Pierri, J. N., Sampson, A. R. \& Lewis, D. A. Decreased glutamic acid decarboxylase67 messenger RNA expression in a subset of prefrontal cortical gamma-aminobutyric acid neurons in subjects with schizophrenia. Arch. Gen. Psychiatry 57, 237-245 (2000).

12. Curley, A. A. et al. Cortical deficits of glutamic acid decarboxylase 67 expression in schizophrenia: Clinical, protein, and cell type-specific features. Am. J. Psychiatry 168, 921-929 (2011).

13. Hashimoto, T. et al. Conserved regional patterns of GABA-related transcript expression in the neocortex of subjects with schizophrenia. Am. J. Psychiatry 165, 479-489 (2008).

14. Glausier, J. R., Kimoto, S., Fish, K. N. \& Lewis, D. A. Lower glutamic acid decarboxylase $65-\mathrm{kDa}$ isoform messenger RNA and protein levels in the prefrontal cortex in schizoaffective disorder but not schizophrenia. Biol. psychiatry 77, 167-176 (2015).

15. Tsubomoto, M. et al. Expression of transcripts selective for GABA neuron subpopulations across the cortical visuospatial working memory network in the healthy state and schizophrenia. Cereb. Cortex 29, 3540-3550 (2019).

16. Thompson Ray, M., Weickert, C. S., Wyatt, E. \& Webster, M. J. Decreased BDNF, trkB-TK+ and GAD67 mRNA expression in the hippocampus of individuals with schizophrenia and mood disorders. J. Psychiatry Neurosci. 36, 195-203 (2011).

17. Keefe, R.S. \& Harvey, P.D. in Novel Antischizophrenia Treatments, Handbook of Experimental Pharmacology (eds. Geyer, M. \& Gross, G.) (Springer, Berlin, Heidelberg, 2012).

18. Gonzalez-Burgos, G. \& Lewis, D. A. GABA neurons and the mechanisms of network oscillations: Implications for understanding cortical dysfunction in schizophrenia. Schizophr. Bull. 34, 944-961 (2008).

19. Yoon, J. H., Grandelis, A. \& Maddock, R. J. Dorsolateral prefrontal cortex GABA concentration in humans predicts working memory load processing capacity. J. Neurosci. 36, 11788-11794 (2016).

20. Addington, A. M. et al. GAD1 (2q31.1), which encodes glutamic acid decarboxylase (GAD 67), is associated with childhood-onset schizophrenia and cortical gray matter volume loss. Mol. Psychiatry 10, 581-588 (2005).

21. Du, J. et al. Comprehensive analysis of polymorphisms throughout GAD1 gene: A family-based association study in schizophrenia. J. Neural Transm. 115 513-519 (2008).

22. Hyde, T. M. et al. Expression of GABA signaling molecules KCC2, NKCC1, and GAD1 in cortical development and schizophrenia. J. Neurosci. 31, 11088-11095 (2011).

23. Straub, R. E. et al. Allelic variation in GAD1 (GAD 67) is associated with schizophrenia and influences cortical function and gene expression. Mol. Psychiatry 12, 854-869 (2007).

24. Giacopuzzi, E. et al. Exome sequencing in schizophrenic patients with high levels of homozygosity identifies novel and extremely rare mutations in the GABA/glutamatergic pathways. PLoS ONE 12, e0182778 (2017).

25. Magri, $C$. et al. A novel homozygous mutation in GAD1 gene described in a schizophrenic patient impairs activity and dimerization of GAD67 enzyme. Sci. Rep. 8, 15470 (2018).

26. Kakizaki, T., Oriuchi, N. \& Yanagawa, Y. GAD65/GAD67 double knockout mice exhibit intermediate severity in both cleft palate and omphalocele compared with GAD67 knockout and VGAT knockout mice. Neuroscience 288, 86-93 (2015).

27. Brown, J. A. et al. Inhibition of parvalbumin-expressing interneurons results in complex behavioral changes. Mol. Psychiatry 20, 1499-1507 (2015).

28. Fujihara, K. et al. Glutamate decarboxylase 67 deficiency in a subset of GABAergic neurons induces schizophrenia-related phenotypes. Neuropsychopharmacolog 40, 2475-2486 (2015).

29. Kolata, S. M. et al. Neuropsychiatric phenotypes produced by GABA reduction in mouse cortex and hippocampus. Neuropsychopharmacology 43, 1445-1456 (2018).
30. Cong, L. et al. Multiplex genome engineering using CRISPR/Cas systems. Science 339, 819-823 (2013).

31. Leo, D. et al. Pronounced hyperactivity, cognitive dysfunctions, and BDNF dysregulation in dopamine transporter knock-out rats. J. Neurosci. 38, 1959-1972 (2018).

32. Bao, D. et al. Preliminary characterization of a leptin receptor knockout rat created by CRISPR/Cas9 system. Sci. Rep. 5, 15942 (2015).

33. Johnson, M. B. et al. Aspm knockout ferret reveals an evolutionary mechanism governing cerebral cortical size. Nature 556, 370-375 (2018).

34. Sato, K. et al. Generation of a nonhuman primate model of severe combined immunodeficiency using highly efficient genome editing. Cell Stem Cell 19, 127-138 (2016).

35. Ellenbroek, B. \& Youn, J. Rodent models in neuroscience research: is it a rat race? Dis. Model Mech. 9, 1079-1087 (2016).

36. Sheikh, S. N., Martin, S. B. \& Martin, D. L. Regional distribution and relative amounts of glutamate decarboxylase isoforms in rat and mouse brain. Neurochem. Int. 35, 73-80 (1999).

37. $\mathrm{Xu}, \mathrm{G}$. et al. Late development of the GABAergic system in the human cerebral cortex and white matter. J. Neuropathol. Exp. Neurol. 70, 841-858 (2011).

38. Miyasaka, Y. et al. CLICK: One-step generation of conditional knockout mice. BMC Genomics 19, 318 (2018).

39. Yanagawa, Y. et al. Structure and alternative promoters of the mouse glutamic acid decarboxylase 67 gene. Biochem. J. 326, 573-578 (1997).

40. Liu, H. et al. Utilization of an intron located polyadenlyation site resulted in four novel glutamate decarboxylase transcripts. Mol. Biol. Rep. 36, 1469-1474 (2009).

41. Suto, T., Kato, D., Obata, H. \& Saito, S. Tropomyosin receptor kinase B receptor activation in the locus coeruleus restores impairment of endogenous analgesia at a late stage following nerve injury in rats. J. Pain. 20, 600-609 (2019).

42. Kawabe, K., Iwasaki, T. \& Ichitani, Y. Repeated treatment with N-methyl-Daspartate antagonists in neonatal, but not adult, rats causes long-term deficits of radial-arm maze learning. Brain Res. 1169, 77-86 (2007).

43. Nunez, J. Morris water maze experiment. J. Vis. Exp. 19, pii 897 (2008).

44. Bubeníková-Valešová, V., Horáček, J., Vrajová, M. \& Höschl, C. Models of schizophrenia in humans and animals based on inhibition of NMDA receptors. Neurosci. Biobehav. Rev. 32, 1014-1023 (2008).

45. Antunes, M. \& Biala, G. The novel object recognition memory: Neurobiology, test procedure, and its modifications. Cogn. Process 13, 93-110 (2012).

46. Eckerman, D. A. Monte Carlo estimation of chance performance for the radial arm maze. Bull. Psychon. Soc. 15, 93-95 (1980).

47. Pallotto, M. \& Deprez, F. Regulation of adult neurogenesis by GABAergic transmission: signaling beyond GABAA-receptors. Front. Cell Neurosci. 8, 166 (2014).

48. Kang, E., Wen, Z., Song, H., Christian, K. M. \& Ming, G. L. Adult neurogenesis and psychiatric disorders. Cold Spring Harb. Perspect. Biol. 8, a019026 (2016).

49. Morris, R. G., Garrud, P., Rawlins, J. N. \& O'Keefe, J. Place navigation impaired in rats with hippocampal lesions. Nature 297, 681-683 (1982).

50. Olton, D. S. \& Papas, B. C. Spatial memory and hippocampal function. Neuropsychologia 17, 669-682 (1979).

51. Richman, C. L., Dember, W. N. \& Kim, P. Spontaneous alternation behavior in animals: A review. Curr. Psychol. 5, 358-391 (1986).

52. Toda, T., Parylak, S. L., Linker, S. B. \& Gage, F. H. The role of adult hippocampal neurogenesis in brain health and disease. Mol. Psychiatry 24, 67-87 (2019).

53. Murray, A. J. et al. Parvalbumin-positive CA1 interneurons are required for spatial working but not for reference memory. Nat. Neurosci. 14, 297-299 (2011).

54. Andrews-Zwilling, Y. et al. Hilar GABAergic interneuron activity controls spatial learning and memory retrieval. PLOS ONE 7, e40555 (2012).

55. Georgiev, D. et al. Cortical gene expression after a conditional knockout of 67 kDa glutamic acid decarboxylase in parvalbumin neurons. Schizophr. Bull. 42, 992-1002 (2016).

56. Tamamaki, N. et al. Green fluorescent protein expression and colocalization with calretinin, parvalbumin, and somatostatin in the GAD67-GFP knock-in mouse. J. Comp. Neurol. 467, 60-79 (2003).

57. Uematsu, M. et al. Quantitative chemical composition of cortical GABAergic neurons revealed in transgenic venus-expressing rats. Cereb. Cortex 18, 315-330 (2008).

58. Kawabe, K. Effects of chronic forced-swim stress on behavioral properties in rats with neonatal repeated MK-801 treatment. Pharmacol. Biochem. Behav. 159, 48-54 (2017). 
59. Sano, W. et al. Enhanced persistency of resting and active periods of locomotor activity in schizophrenia. PLOS ONE 7, e43539 (2012).

60. Parks, C. L., Robinson, P. S., Sibille, E., Shenk, T. \& Toth, M. Increased anxiety of mice lacking the serotonin1A receptor. Proc. Natl Acad. Sci. USA 95 10734-10739 (1998).

61. Seibenhener, M. L. \& Wooten, M. C. Use of the open field maze to measure locomotor and anxiety-like behavior in mice. J. Vis. Exp. 96, e52434 (2015)

62. Miyata, S. et al. Loss of glutamate decarboxylase 67 in somatostatin-expressing neurons leads to anxiety-like behavior and alteration in the akt/gsk3 $\beta$ signaling pathway. Front. Behav. Neurosci. 13, 131 (2019).

63. Bubser, M., Keseberg, U., Notz, P. K. \& Schmidt, W. J. Differential behavioural and neurochemical effects of competitive and non-competitive NMDA receptor antagonists in rats. Eur. J. Pharmacol. 229, 75-82 (1992).

64. Kawabe, K. \& Miyamoto, E. Effects of neonatal repeated MK-801 treatment on delayed nonmatching-to-position responses in rats. Neuroreport 19,969-973 (2008).

65. Uehara, T. et al. Neonatal exposure to MK-801, an N-methyl-D-aspartate receptor antagonist, enhances methamphetamine-induced locomotion and disrupts sensorimotor gating in pre- and postpubertal rats. Brain Res. 1352, 223-230 (2010).

66. Wang, C. Z., Yang, S. F., Xia, Y. \& Johnson, K. M. Postnatal phencyclidine administration selectively reduces adult cortical parvalbumin-containing interneurons. Neuropsychopharmacology 33, 2442-2455 (2008).
67. Lema Tomé, C. M. et al. Decline in age-dependent, MK801-induced injury coincides with developmental switch in parvalbumin expression: Somatosensory and motor cortex. Dev. Psychobiol. 50, 665-679 (2008).

68. Heldt, S. A., Green, A. \& Ressler, K. J. Prepulse inhibition deficits in GAD65 knockout mice and the effect of antipsychotic treatment. Neuropsychopharmacology 29, 1610-1619 (2004).

69. Wu, Q., Boyle, M. P. \& Palmiter, R. D. Loss of GABAergic signaling by AgRP neurons to the parabrachial nucleus leads to starvation. Cell 137, 1225-1234 (2009).

70. Kim, E. R. et al. Hypothalamic Non-AgRP, Non-POMC GABAergic neurons are required for postweaning feeding and NPY hyperphagia. J. Neurosci. 35, 10440-10450 (2015).

71. Tsuji, M. et al. A new case of GABA transaminase deficiency facilitated by proton MR spectroscopy. J. Inherit. Metab. Dis. 33, 85-90 (2010).

72. Louro, P. et al. Phenotyping GABA transaminase deficiency: a case description and literature review. J. Inherit. Metab. Dis. 39, 743-747 (2016).

73. Koenig, M. K. et al. Phenotype of GABA-transaminase deficiency. Neurology $\mathbf{8 8}$ 1919-1924 (2017).

74. Anderson, R. A. \& Mitchell, R. Effects of y-aminobutyric acid receptor agonists on the secretion of growth hormone, luteinizing hormone, adrenocorticotrophic hormone and thyroid-stimulating hormone from the rat pituitary gland in vitro. J. Endocrinol. 108, 1-8 (1986).

75. Ripke, S. et al. Biological insights from 108 schizophrenia-associated genetic loci. Nature 511, 421-427 (2014). 